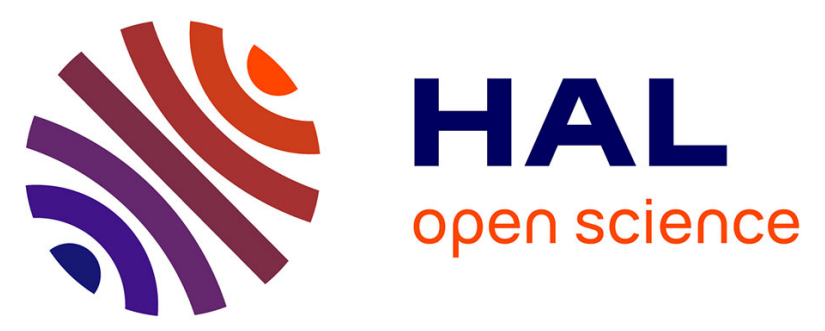

\title{
Four Highly Dispersed Millisecond Pulsars Discovered in the Arecibo PALFA Galactic Plane Survey
}

F. Crawford, K. Stovall, A. G. Lyne, B. W. Stappers, D. J. Nice, I. H. Stairs, P. Lazarus, J. W. T. Hessels, P. C. C. Freire, B. Allen, et al.

\section{- To cite this version:}

F. Crawford, K. Stovall, A. G. Lyne, B. W. Stappers, D. J. Nice, et al.. Four Highly Dispersed Millisecond Pulsars Discovered in the Arecibo PALFA Galactic Plane Survey. The Astrophysical Journal, 2012, 757 (90), 10 p. 10.1088/0004-637X/757/1/90 . insu-01266087

\section{HAL Id: insu-01266087 https://hal-insu.archives-ouvertes.fr/insu-01266087}

Submitted on 19 May 2016

HAL is a multi-disciplinary open access archive for the deposit and dissemination of scientific research documents, whether they are published or not. The documents may come from teaching and research institutions in France or abroad, or from public or private research centers.
L'archive ouverte pluridisciplinaire HAL, est destinée au dépôt et à la diffusion de documents scientifiques de niveau recherche, publiés ou non, émanant des établissements d'enseignement et de recherche français ou étrangers, des laboratoires publics ou privés.

\section{(1) (1) $\$$}

Distributed under a Creative Commons Attribution - NonCommercial - NoDerivatives $\mid 4.0$ 


\title{
FOUR HIGHLY DISPERSED MILLISECOND PULSARS DISCOVERED IN THE ARECIBO PALFA GALACTIC PLANE SURVEY
}

\author{
F. Crawford ${ }^{1}$, K. Stovall ${ }^{2,3}$, A. G. Lyne ${ }^{4}$, B. W. Stappers ${ }^{4}$, D. J. Nice ${ }^{5}$, I. H. Stairs ${ }^{6}$, P. Lazarus ${ }^{7,8}$, J. W. T. Hessels $^{9,10}$, \\ P. C. C. Freire $^{8}$, B. Allen ${ }^{11,12,13}$, N. D. R. Bhat ${ }^{14}$, S. Bogdanov ${ }^{15}$, A. Brazier ${ }^{16}$, F. Camilo ${ }^{14,17}$, D. J. Champion ${ }^{8}$, \\ S. Chatterjee ${ }^{16}$, I. Cognard ${ }^{18}$, J. M. Cordes ${ }^{16}$, J. S. Deneva ${ }^{17}$, G. Desvignes ${ }^{8,18}$, F. A. Jenet ${ }^{2}$, V. M. Kaspi ${ }^{7}$, B. Knispel ${ }^{11,12}$,

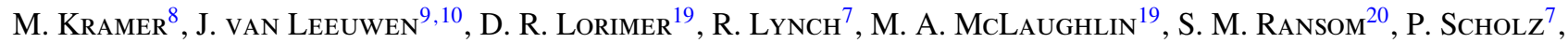 \\ X. Siemens ${ }^{13}$, And A. Venkataraman ${ }^{17}$ \\ ${ }^{1}$ Department of Physics and Astronomy, Franklin and Marshall College, P.O. Box 3003, Lancaster, PA 17604, USA; fcrawfor@fandm.edu \\ ${ }^{2}$ Center for Gravitational Wave Astronomy, University of Texas at Brownsville, Brownsville, TX 78520, USA \\ ${ }^{3}$ Department of Physics and Astronomy, University of Texas at San Antonio, San Antonio, TX 78249, USA \\ ${ }^{4}$ Jodrell Bank Centre for Astrophysics, University of Manchester, Manchester M13 9PL, UK \\ ${ }^{5}$ Department of Physics, Lafayette College, Easton, PA 18042, USA \\ ${ }^{6}$ Department of Physics and Astronomy, University of British Columbia, 6224 Agricultural Road, Vancouver, BC V6T 1Z1, Canada \\ ${ }^{7}$ Department of Physics, McGill University, 3600 University Street, Montreal, QC H3A 2T8, Canada \\ ${ }^{8}$ Max-Planck-Institut für Radioastronomie, auf dem Huegel 69, D-53121 Bonn, Germany \\ ${ }_{9}^{9}$ ASTRON, The Netherlands Institute for Radio Astronomy, Postbus 2, 7990-AA Dwingeloo, The Netherlands \\ ${ }^{10}$ Astronomical Institute “Anton Pannekoek," University of Amsterdam, Science Park 904, 1098-XH Amsterdam, The Netherlands \\ ${ }^{11}$ Albert-Einstein-Institut, Max-Planck-Institut für Gravitationsphysik, D-30167 Hannover, Germany \\ ${ }_{12}$ Institut für Gravitationsphysik, Leibniz Universität Hannover, D-30167 Hannover, Germany \\ ${ }^{13}$ Physics Department, University of Wisconsin at Milwaukee, Milwaukee, WI 53211, USA \\ ${ }^{14}$ Center for Astrophysics and Supercomputing, Swinburne University, Hawthorn, Victoria 3122, Australia \\ ${ }^{15}$ Columbia Astrophysics Laboratory, Columbia University, New York, NY 10027, USA \\ ${ }^{16}$ Astronomy Department, Cornell University, Ithaca, NY 14853, USA \\ ${ }_{17}$ Arecibo Observatory, HC3 Box 53995, Arecibo, PR 00612, USA \\ ${ }^{18}$ Laboratoire de Physique et Chimie de l'Environnement et de l'Espace, LPC2E, CNRS et Université d'Orléans, \\ and Station de radioastronomie de Nançay, Observatoire de Paris, F-18330 Nancay, France \\ ${ }^{19}$ Department of Physics, West Virginia University, Morgantown, WV 26506, USA \\ ${ }^{20}$ National Radio Astronomy Observatory, 520 Edgemont Road, Charlottesville, VA 22903, USA \\ Received 2012 May 21; accepted 2012 August 6; published 2012 September 6
}

\begin{abstract}
We present the discovery and phase-coherent timing of four highly dispersed millisecond pulsars (MSPs) from the Arecibo PALFA Galactic plane survey: PSRs J1844+0115, J1850+0124, J1900+0308, and J1944+2236. Three of the four pulsars are in binary systems with low-mass companions, which are most likely white dwarfs, and which have orbital periods on the order of days. The fourth pulsar is isolated. All four pulsars have large dispersion measures $\left(\mathrm{DM}>100 \mathrm{pc} \mathrm{cm}^{-3}\right)$, are distant $(\gtrsim 3.4 \mathrm{kpc})$, faint at $1.4 \mathrm{GHz}(\lesssim 0.2 \mathrm{mJy})$, and are fully recycled (with spin periods $P$ between 3.5 and $4.9 \mathrm{~ms}$ ). The three binaries also have very small orbital eccentricities, as expected for tidally circularized, fully recycled systems with low-mass companions. These four pulsars have DM $/ P$ ratios that are among the highest values for field MSPs in the Galaxy. These discoveries bring the total number of confirmed MSPs from the PALFA survey to 15. The discovery of these MSPs illustrates the power of PALFA for finding weak, distant MSPs at low-Galactic latitudes. This is important for accurate estimates of the Galactic MSP population and for the number of MSPs that the Square Kilometer Array can be expected to detect.
\end{abstract}

Key words: pulsars: general - pulsars: individual (PSR J1844+0115, PSR J1850+0124, PSR J1900+0308, PSR J1944+2236) - surveys

\section{INTRODUCTION}

The PALFA survey is an ongoing, large-scale pulsar survey of the Galactic plane that uses the Arecibo $305 \mathrm{~m}$ radio telescope and the Arecibo L-Band Feed Array (ALFA) 7-beam multibeam receiver (Cordes et al. 2006). It is one of Arecibo's key science projects, and it will ultimately cover the entire Arecibo-visible sky within $5^{\circ}$ of the Galactic plane (longitudes of $32^{\circ} \lesssim l \lesssim 77^{\circ}$ and $168^{\circ} \lesssim l \lesssim 214^{\circ}$ ). PALFA observes at relatively high observing frequencies $(1220-1520 \mathrm{MHz})$ in order to mitigate the deleterious effects that interstellar dispersion and scattering have on the detection of distant pulsars at low Galactic latitudes. In this sense, the PALFA survey is similar to the highly successful Parkes Multibeam Pulsar Survey (PMPS; Manchester et al. 2001), but with increased time and frequency resolution, such as those of the current Parkes HTRU survey (Keith et al. 2010).

Compared to past Arecibo surveys, PALFA explores a far larger spatial volume due to its high time and frequency resolution, enabling discovery of faint, highly dispersed millisecond pulsars (MSPs) ${ }^{21}$ in the Galactic plane, which tend to be at larger distances. Since the majority of MSPs have binary companions ( $~ 80 \%$; Lorimer 2008$)$, they are often interesting test cases for studies of exotic binary stellar evolution (e.g., Archibald et al. 2009; Freire et al. 2011). The unsurpassed sensitivity of the Arecibo telescope is highly advantageous for detecting binary MSPs because the PALFA pointing dwell times are only a small fraction (less than $10 \%$ ) of the orbital periods of all known binary radio pulsars. In this regime, linear acceleration searches are highly effective at recovering Doppler-smeared periodicities (Johnston \& Kulkarni 1991).

The discovery of PSR J1903+0327 by Champion et al. (2008) is an excellent illustration of the PALFA survey's sensitivity to

\footnotetext{
21 An MSP typically refers to a (partially or fully) recycled pulsar having a small surface magnetic field strength $\left(B \lesssim 10^{10} \mathrm{G}\right)$ and a large characteristic age $\left(\gtrsim 10^{9} \mathrm{yr}\right)$.
} 
highly dispersed pulsars. PSR J1903+0327 has a spin period of only $2.15 \mathrm{~ms}$ and has the highest dispersion measure (DM) of all known completely recycled Galactic MSPs, $297 \mathrm{pc} \mathrm{cm}^{-3}$. It occupies a region of DM-period phase space that has previously been unexplored for pulsars in the Galactic field. For example, of the 90 recycled Galactic field radio pulsars currently listed in the ATNF catalog (apart from the PALFA discoveries) ${ }^{22}$ with spin periods $P<25 \mathrm{~ms}$, only 9 have DM $>100 \mathrm{pc} \mathrm{cm}^{-3}$, and only 14 have DM-derived distances listed in the catalog that are greater than $3 \mathrm{kpc}$.

One of the main motivations for finding distant, highly dispersed MSPs is to determine a more complete census of the Galactic MSP population, which is currently biased by the large number of nearby $(\lesssim 2 \mathrm{kpc})$ sources-especially given the recent discoveries of more than 40 generally nearby MSPs through targeted searches of Fermi gamma-ray sources (e.g., Ransom et al. 2011). MSPs are the longest-living active manifestations of neutron stars, with active lives hundreds to thousands of times longer than those of normal pulsars, magnetars, or accreting neutron stars. Hence, they give valuable insight into the Galactic neutron star population and binary stellar evolution in particular. The planned Square Kilometer Array (SKA; e.g., Carilli \& Rawlings 2004) should be able to detect a large fraction of the MSPs in the Galaxy, and finding high-DM MSPs beforehand will help tell us how many to expect. Furthermore, MSPs are excellent probes of the interstellar medium, and the discovery of more distant MSPs whose signals likely pass through multiple scattering screens opens new possibilities (and challenges) in this area. For instance, scattering measurements of large-DM MSPs can be used to compare the observed effects of scattering on timing behavior with predictions of those effects.

MSPs can also be used as high-precision astronomical clocks. Stable MSPs form the basis of the pulsar timing efforts of the International Pulsar Timing Array consortium to detect longperiod gravitational radiation from observations of pulsar timing residuals (Jenet et al. 2005; Hobbs et al. 2010). The recent precision mass measurements of PSR J1903+0327 (Freire et al. 2011) and PSR J1614-2230 (Demorest et al. 2010) indicate that MSPs can have masses well above the Chandrasekhar mass, and mass constraints from new MSPs will continue to map out the MSP mass distribution. The PALFA pulsar PSR J1949+3106, which may also have a higher mass than the Chandrasekhar mass (Deneva et al. 2012), is an example where future mass measurements may prove important. Although high-DM MSPs have timing precision problems associated with interstellar scattering in addition to their being generally radio-faint, future observations with next generation instruments like the SKA may be able to mitigate these factors. Precision measurement of NS masses (e.g., Demorest et al. 2010) and the measurement of ultra-high spin rates (e.g., Lattimer \& Prakash 2007; Hessels et al. 2006) can also rule out some high-density NS equations of state.

In this paper, we report the discovery and follow-up timing of four highly dispersed MSPs from the PALFA survey: PSRs J1844+0115, J1850+0124, J1900+0308, and J1944+2236. These MSPs all have DMs that are in the top 5\% of non-PALFA field radio MSPs. Three of the four pulsars are in binary systems with low-mass companions. These discoveries bring the number of PALFA recycled pulsar discoveries to 15 , including PSR J1903+0327 (Champion et al. 2008), PSRs J1949+3106

\footnotetext{
22 We also exclude the high-DM MSPs in globular clusters, because these are found by targeted searches conducted with very long integration times and at even higher observing frequencies (generally $\sim 2 \mathrm{GHz}$ ).
}

and J1955+2427 (Deneva et al. 2012), and the partially recycled PSRs J2007+2722 and J1952+2630 (Knispel et al. 2010, 2011). The six additional recycled pulsars that have been confirmed in the survey need to be timed further to establish their rotational and orbital characteristics. These six pulsars will be published in forthcoming papers and are not considered further here. In Section 2, we describe the discovery and follow-up observations of the four pulsars, and we present their phase-coherent timing solutions. In Section 3, we discuss our results, and in Section 4 we present our conclusions.

\section{DISCOVERY, TIMING, AND POLARIMETRY OBSERVATIONS}

\subsection{Discovery}

Until 2009 February, survey data for the PALFA survey were recorded with the Wideband Arecibo Pulsar Processor (WAPP) auto-correlation spectrometers (Dowd et al. 2000; Cordes et al. 2006). ${ }^{23}$ The WAPP backends had 3-level sampling and provided $100 \mathrm{MHz}$ of bandwidth for each of the 7 ALFA beams, centered on a sky frequency of $1.4 \mathrm{GHz}$ and split into 256 lags (channels). These lags were sampled at $64 \mu \mathrm{s}$ and each survey pointing was observed for $268 \mathrm{~s}$. The four pulsars presented here were discovered in data taken with this setup.

PALFA survey data are archived at the Cornell University Center for Advanced Computing, where they are processed locally using custom search software and are also staged for transport to other PALFA consortium sites. The data are processed at these sites with dedicated computer clusters that use a search pipeline based on the PRESTO software suite (Ransom 2001; Ransom et al. 2002). ${ }^{24}$ Another analysis pipeline is also used which employs the huge volunteer computing resources provided by the "Einstein@Home" project ${ }^{25}$ to search the survey data for binary systems with orbital periods as short as 11 minutes (Knispel et al. 2011). Results from the PRESTO processing (including candidate plots and associated information) are uploaded to a collaborative Web portal (www.cyberska.org), where interactive applications are used to visually inspect and rate selected candidates.

The four MSPs presented here were all discovered with the PRESTO search pipeline in survey pointings taken at different epochs (see Table 1 for the discovery observation MJDs). Figure 1 shows the integrated pulse profiles for the four MSPs obtained by phase aligning and adding the folded profiles from Arecibo $1.4 \mathrm{GHz}$ observations. All of the integrated profiles in Figure 1 have moderate widths $(\sim 15 \%-30 \%$ of the pulse period). In two cases (PSRs J1844+0115 and J1850+0124), the profiles are clearly single peaked. For PSR J1900+0308, there is some indication of a weaker leading component (and possibly also a trailing component). PSR J1944+2236 may have a hint of a secondary peak close to the center of the profile. None of the profiles shows any clear evidence of scattering, which is not surprising: according to the NE2001 model of Cordes \& Lazio (2002), the pulse scattering time for these pulsars is expected to be small in all cases at $1.4 \mathrm{GHz}(\lesssim 0.04 \mathrm{~ms})$. Bhat et al. (2004) have noted that in some cases pulse scattering times can be underestimated by the NE2001 model by up to an order

\footnotetext{
23 Since 2009, the survey has been recording data using the Jeff Mock spectrometers, which are polyphase filter banks that provide better radio frequency interference rejection as well as increased bandwidth compared to the earlier WAPP survey data.

24 http://www.cv.nrao.edu/ sransom/presto

25 http://einstein.phys.uwm.edu
} 
Table 1

Timing Parameters for Four MSPs

\begin{tabular}{|c|c|c|c|c|}
\hline PSR & $\mathrm{J} 1844+0115$ & $\mathrm{~J} 1850+0124$ & $\mathrm{~J} 1900+0308$ & $\mathrm{~J} 1944+2236$ \\
\hline Right ascension (J2000) & $18: 44: 40.5474(3)$ & 18:50:01.0139(8) & 19:00:50.5548(3) & 19:44:01.0707(11) \\
\hline Declination (J2000) & $+01: 15: 34.974(11)$ & $+01: 24: 34.61(3)$ & $+03: 08: 24.079(13)$ & $+22: 36: 22.62(2)$ \\
\hline Spin frequency, $f\left(s^{-1}\right)$ & 238.91757323111(15) & $280.9175173300(4)$ & $203.6975581461(2)$ & $276.3963275801(5)$ \\
\hline Frequency derivative, $\dot{f}\left(s^{-2}\right)$ & $-6.12(12) \times 10^{-16}$ & $-8.6(4) \times 10^{-16}$ & $-2.45(9) \times 10^{-16}$ & $-5.7(4) \times 10^{-16}$ \\
\hline Dispersion measure, DM $\left(\mathrm{pc} \mathrm{cm}^{-3}\right)$ & $148.22(2)$ & $118.89(5)$ & $249.898(11)$ & $185.45(12)$ \\
\hline Orbital period, $P_{b}(\mathrm{~d})$ & $50.6458881(11)$ & $84.949858(4)$ & $12.47602144(10)$ & $\cdots$ \\
\hline Time of periastron passage, $T_{0}(\mathrm{MJD})^{\mathrm{a}}$ & $55409.21(4)$ & $55241.3(3)$ & $\cdots$ & $\cdots$ \\
\hline Projected semimajor axis, $x(\mathrm{~s})^{\mathrm{b}}$ & $14.173495(9)$ & $34.00102(2)$ & $6.716377(4)$ & $\cdots$ \\
\hline Longitude of periastron, $\omega(\mathrm{deg})^{\mathrm{a}}$ & $189.6(3)$ & $98.8(11)$ & $\cdots$ & $\cdots$ \\
\hline Eccentricity, $e$ & $2.578(11) \times 10^{-4}$ & $6.90(12) \times 10^{-5}$ & $<3.3 \times 10^{-6}$ & $\cdots$ \\
\hline Time of ascending node, $T_{\mathrm{asc}}(\mathrm{MJD})^{\mathrm{c}}$ & $55382.532417(5)$ & $55217.963824(11)$ & $55304.460562(3)$ & $\cdots$ \\
\hline$\epsilon_{1}=e \sin \omega^{\mathrm{c}}$ & $-4.32(13) \times 10^{-5}$ & $6.82(12) \times 10^{-5}$ & $<3.0 \times 10^{-6}$ & $\cdots$ \\
\hline$\epsilon_{2}=e \cos \omega^{\mathrm{c}}$ & $-2.542(11) \times 10^{-4}$ & $-1.05(13) \times 10^{-5}$ & $<3.1 \times 10^{-6}$ & $\cdots$ \\
\hline Period, $P(\mathrm{~ms})$ & $4.185543936664(3)$ & $3.559763768043(5)$ & $4.909239016417(4)$ & $3.617993078111(6)$ \\
\hline Period derivative, $\dot{P}$ & $1.07(2) \times 10^{-20}$ & $1.09(5) \times 10^{-20}$ & $5.9(2) \times 10^{-21}$ & $7.5(5) \times 10^{-21}$ \\
\hline Mass function $\left(M_{\odot}\right)$ & $0.001191860(2)$ & $0.005848349(13)$ & $0.002089949(4)$ & $\cdots$ \\
\hline Companion mass $\left(M_{\odot}\right)^{\mathrm{d}}$ & $>0.14$ & $>0.25$ & $>0.17$ & $\cdots$ \\
\hline Galactic longitude, $l$ (deg) & 33.28 & 34.02 & 36.79 & 58.90 \\
\hline Galactic latitude, $b$ (deg) & +2.08 & +0.96 & -0.66 & -0.66 \\
\hline Surface magnetic field, $B(\mathrm{G})^{\mathrm{e}}$ & $2.1 \times 10^{8}$ & $2.0 \times 10^{8}$ & $1.7 \times 10^{8}$ & $1.7 \times 10^{8}$ \\
\hline Spin-down luminosity, $\dot{E}\left(\mathrm{erg} \mathrm{s}^{-1}\right)^{\mathrm{e}}$ & $5.8 \times 10^{33}$ & $9.6 \times 10^{33}$ & $2.0 \times 10^{33}$ & $6.4 \times 10^{33}$ \\
\hline Characteristic age, $\tau_{c}(\mathrm{Gyr})^{\mathrm{e}}$ & 6.2 & 5.2 & 13.0 & 7.5 \\
\hline Distance, $d(\mathrm{kpc})^{\mathrm{f}}$ & 3.9 & 3.4 & 5.8 & 6.5 \\
\hline Distance from Galactic plane, $|z|,(\mathrm{kpc})^{\mathrm{g}}$ & 0.14 & 0.06 & 0.07 & 0.08 \\
\hline $1400 \mathrm{MHz}$ flux density, $S_{1400}(\mathrm{mJy})$ & $\sim 0.1$ & $\sim 0.2$ & $\sim 0.2$ & $\lesssim 0.1$ \\
\hline $1400 \mathrm{MHz}$ radio luminosity, $L_{1400}\left(\mathrm{mJy} \mathrm{kpc}^{2}\right)^{\mathrm{h}}$ & $\sim 1.5$ & $\sim 2.3$ & $\sim 6.7$ & $\lesssim 4.2$ \\
\hline Discovery observation MJD & 53493 & 54555 & 53656 & 53647 \\
\hline Timing epoch (MJD) & 55383 & 55383 & 55255 & 55415 \\
\hline TOA range (MJD) & $55152-55914$ & $55153-55915$ & $54907-55927$ & $55222-55926$ \\
\hline Timing span (days) & 762 & 762 & 1020 & 704 \\
\hline Number of points in timing fit (Jodrell/Arecibo) & $116 / 7$ & $110 / 7$ & $94 / 136$ & $77 / 8$ \\
\hline Characteristic TOA residual $(\mu \mathrm{s})$ (Jodrell/Arecibo) & $40 / 10$ & $100 / 10$ & $80 / 10$ & $80 / 10$ \\
\hline TOA error scale factor (Jodrell/WAPP/Mock) ${ }^{\mathrm{i}}$ & $1.20 / 1.00 / \cdots$ & $2.03 / 1.00 / \cdots$ & $1.09 / 1.25 / 1.45$ & $1.87 / \cdots / 1.85$ \\
\hline Weighted rms post-fit residual ( $\mu \mathrm{s})$ & 25.4 & 51.6 & 15.8 & 66.1 \\
\hline
\end{tabular}

Notes. Figures in parentheses are uncertainties in the last digit quoted and are twice the formal errors from the TEMPO timing solution.

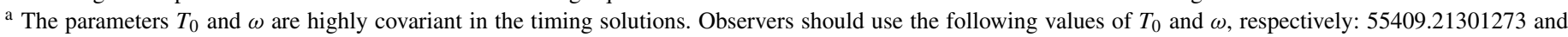
189.6504286222 for PSR J1844+0115; 55241.26871848 and 98.7613423916 for PSR J1850+0124.

b $x=a \sin i / c$ where $a$ is the semimajor axis and $i$ is the orbital inclination angle.

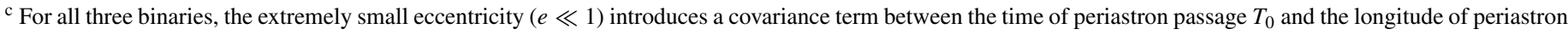

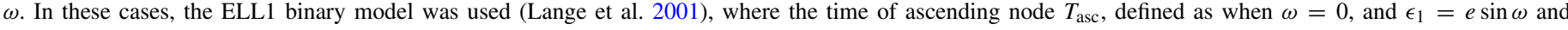
$\epsilon_{2}=e \cos \omega$ are fit instead. $T_{0}, e$, and $\omega$ are derived from this.

d Assumes an inclination angle $i=90^{\circ}$ and a pulsar mass of 1.35 solar masses.

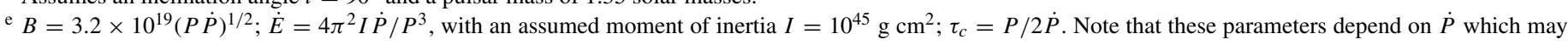
be affected by the Shklovskii effect.

${ }^{\mathrm{f}}$ From the NE2001 DM-distance model of Cordes \& Lazio (2002).

g $|z|=d \sin |b|$.

${ }^{\text {h }} L_{1400}=S_{1400} d^{2}$.

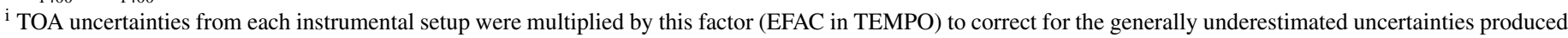
by TEMPO (see the text for justification).

of magnitude (see their Figure 6). However, even if this were the case here, the scattering time would still be $\lesssim 10 \%$ of the pulse period in all cases. At the large DMs of these pulsars, any flux variability would likely be from intrinsic mechanisms rather than scintillation. We see no significant variability or other intermittency (e.g., eclipsing effects) in the observations.

\subsection{Timing}

Soon after the discovery of these pulsars, follow-up timing observations began with the Arecibo telescope and the Lovell telescope at the Jodrell Bank observatory. The Arecibo observa- tions used several systems: the ALFA receiver and single-pixel L-wide receivers, with data recorded with either the WAPPs or the Mock spectrometer.

For the Jodrell Bank observations, dual-polarization cryogenic receivers covered $384 \mathrm{MHz}$ of bandwidth centered at $1520 \mathrm{MHz}$. The bandwidth was split into $0.5 \mathrm{MHz}$ channels in a digital filter bank, and each channel was sampled to provide 1024 samples per pulsar period. The effective sampling time for our four pulsars was therefore a few $\mu$ s and depended on the pulsar period. Each pulsar was observed for between 2400 and $7200 \mathrm{~s}$ per timing session, depending on the pulsar. The sampled data in each channel were folded at the topocentric pulsar 

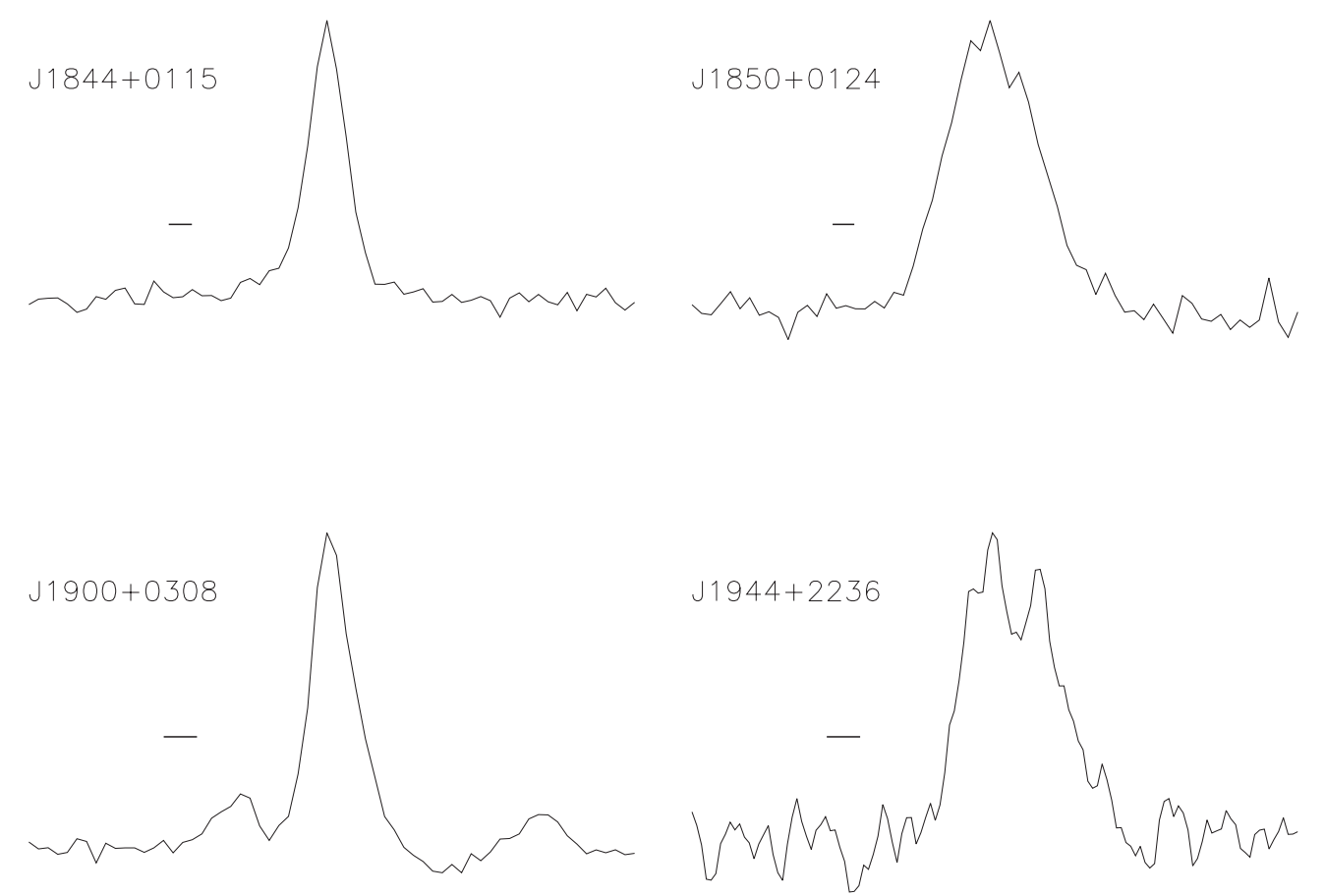

Figure 1. Integrated 1.4 GHz pulse profiles for four MSPs. The pulse profiles were constructed by phase aligning and adding between 30 and 50 minutes of Arecibo observations for each pulsar. A combination of WAPP and Mock data was used for the profiles. In all cases, 64 profile bins were used, except for PSR J1944+2236 where 128 bins were used. One full period is shown in each case. The vertical axis is arbitrary and has been scaled so that all profiles have the same maximum value. The horizontal bars indicate the amount of dispersion smearing within channels for Arecibo observations taken with the WAPPs.

period in each case (this was predicted by an ephemeris), and the channels were subsequently dedispersed at the ephemeris $\mathrm{DM}$ and summed.

For the Arecibo observations, the WAPP systems were used which covered two adjacent $50 \mathrm{MHz}$ bands. Each WAPP had 512 lags and a sampling time of $64 \mu \mathrm{s}$. The pulsars were typically observed for $600 \mathrm{~s}$ in each observation. For PSR J1900+0308, some observations used the Mock spectrometers (see, e.g., Deneva et al. 2012).

The folded profiles from the Arecibo observations were phase aligned and summed to produce a high signal-to-noise pulse template for each pulsar (see Figure 1). Each observation was dedispersed and folded with the latest timing ephemeris, and folded profiles from each observation were cross-correlated in the Fourier domain with the profile template to obtain timesof-arrival (TOAs) with uncertainties. A separate pulse template was produced from the Jodrell Bank observations and this was used to similarly produce the Jodrell Bank TOAs. Table 1 shows the number of TOAs generated from Arecibo and Jodrell Bank with their typical rms values.

We fit the resulting TOAs using the TEMPO software package $^{26}$ and standard pulsar timing procedures (see, e.g., Freire et al. 2011 for details). Initial phase connections for the pulsars were obtained from the Jodrell Bank observations alone, and these were subsequently supplemented with Arecibo TOAs at a variety of frequencies. The Arecibo TOAs allowed us to constrain the DM for each pulsar. All three binaries have small values for the product of orbital eccentricity and projected semimajor axis (see Table 1), so we used the ELL1 binary orbital model of Lange et al. (2001) for the timing solutions.

Figures 2 and 3 show the timing residuals for the four MSPs as a function of both date and orbital phase (except for

\footnotetext{
26 http://tempo.sourceforge.net. Note that we also obtained consistent results
} with the TEMPO2 package (Hobbs et al. 2006).
PSR J1944+2236, which is isolated). As commonly happens in MSP timing, we found the formal TOA uncertainties in some data sets to be underestimated by up to a factor of 2.0. We scaled the TOA uncertainties by a common factor for each observational setup (telescope and backend combination) in order to make the reduced $\chi^{2}$ of each TOA subset equal to unity, and we used the scaled uncertainties to weight the data in the timing fit. Increasing all uncertainties by a factor until the normalized $\chi^{2}$ is one is a conservative step, which produces less precise timing parameters, but ones that are justified by the actual rms of the TOAs available. The scaling factors are listed in Table 1. There are no obvious systematic trends seen in the timing residuals, and the typical residual rms values from the timing solutions are less than $2 \%$ of the pulse period in each case (Table 1). These results indicate that the model is adequately describing the TOAs, with no significant unmodeled effects present.

The full timing solutions for the four MSPs are presented in Table 1, with the listed uncertainties representing twice the formal uncertainties produced by TEMPO. Apart from the directly measured astrometric, spin, and orbital parameters, derived physical parameters are also included. The physical parameters assume a pure magnetic dipole spin-down. Estimated distances and luminosities for the pulsars are also calculated and presented in the table. Note that the Shklovskii effect (Shklovskii 1970) and accelerations from the Galactic potential are not accounted for in the measured values of $\dot{P}$. Until proper motions can be measured (see discussion below), we cannot correct for this effect.

\subsection{Polarimetry}

All four pulsars were also observed in a set of $1.4 \mathrm{GHz}$ Arecibo polarimetry observations in order to measure polarization characteristics in the pulse profiles and to obtain rotation measures (RMs) and calibrated flux density estimates 

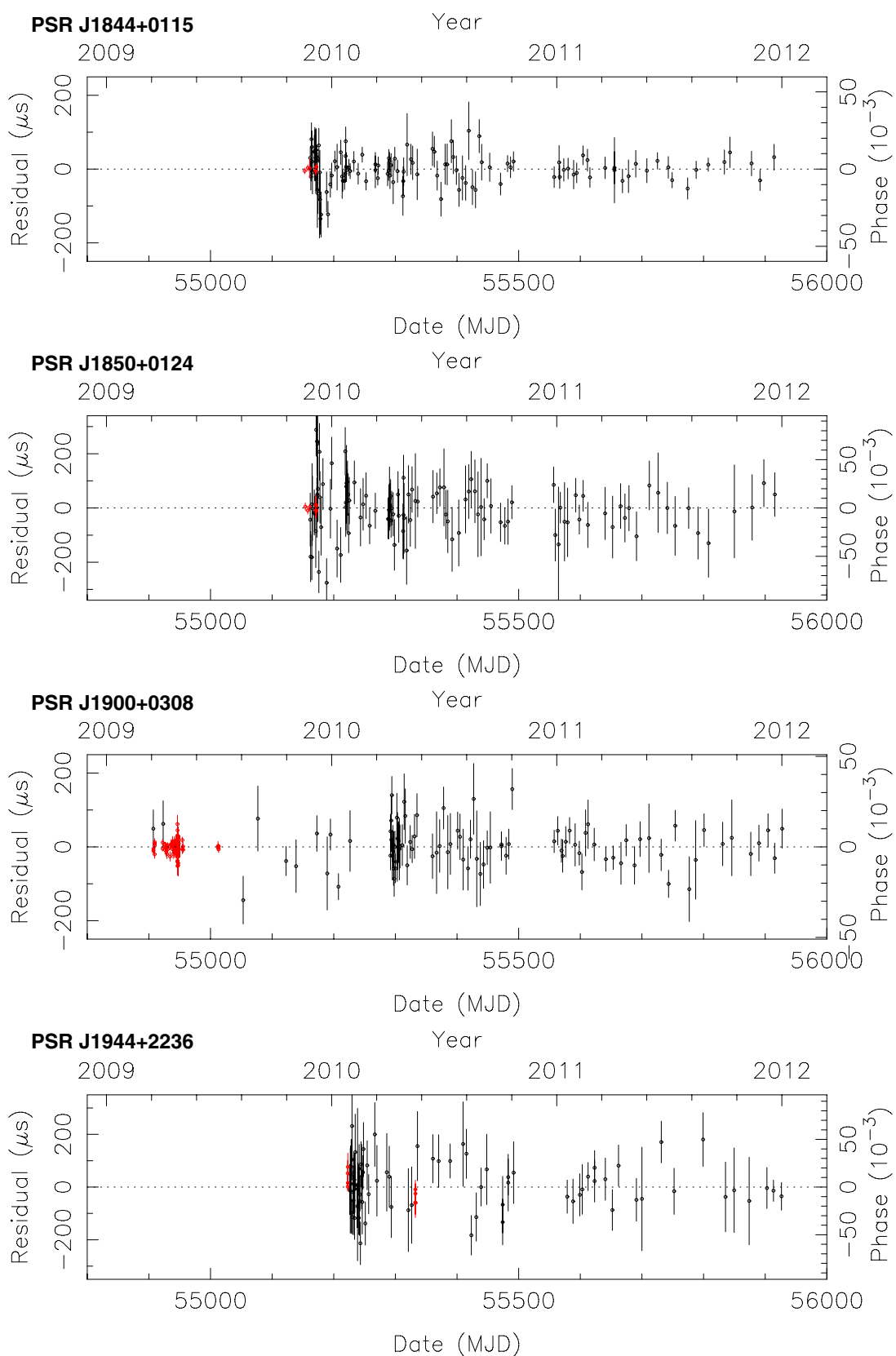

Figure 2. Timing residuals for four MSPs as a function of date. Black points indicate Jodrell Bank TOAs and red points indicate Arecibo TOAs. The error bars shown were produced for each individual TOA.

for the pulsars. Each pulsar was observed for between 5 and 15 minutes at a center frequency of $1412 \mathrm{MHz}$ using the Arecibo signal processor (ASP) backend (Demorest 2007). A total bandwidth of $24 \mathrm{MHz}$ was split into 6 channels, each of $4 \mathrm{MHz}$, and the data were coherently dedispersed and folded during the observation. The data were then processed using PSRCHIVE tools (Hotan et al. 2004). The resulting calibrated data files contained full Stokes parameters for each pulse profile bin. The Stokes parameters were converted to total intensity, linearly and circularly polarized intensity, and position angle (P.A.) values.

We attempted to search for the RM for each pulsar in order to correct the data for Faraday rotation. We used trial RM values to see which trial produced the maximum linear polarization when the frequency channels were summed. This would indicate that the correct RM had been found. We were unable to find a reliable RM estimate in any of the four cases. Figure 4 shows the polarization profiles for the four pulsars without any Faraday rotation correction. None of the pulsars shows clearly significant polarization, with the possible exception of PSR J1900+0308, which may have an excess of right-handed circular polarization across the on-pulse bins. None of the pulsars has a linearly polarized intensity that is significant enough for reliable P.A. measurements across the on-pulse bins (in fact, only PSR J1844+0115 has any measurable P.A.s at all). Faraday smearing across the band is unlikely to be a significant factor in the small measured polarized signal. For RMs as high as a few hundred $\mathrm{rad} \mathrm{m}^{-2}$, the uncorrected Faraday rotation would reduce the linear polarization by only a few percent.

The calibrated files were also used to determine $1.4 \mathrm{GHz}$ flux densities for the pulsars (Table 1). In all cases except PSR J1944+2236, we obtained flux density estimates, but only one digit of precision is quoted owing to the significant uncertainties 

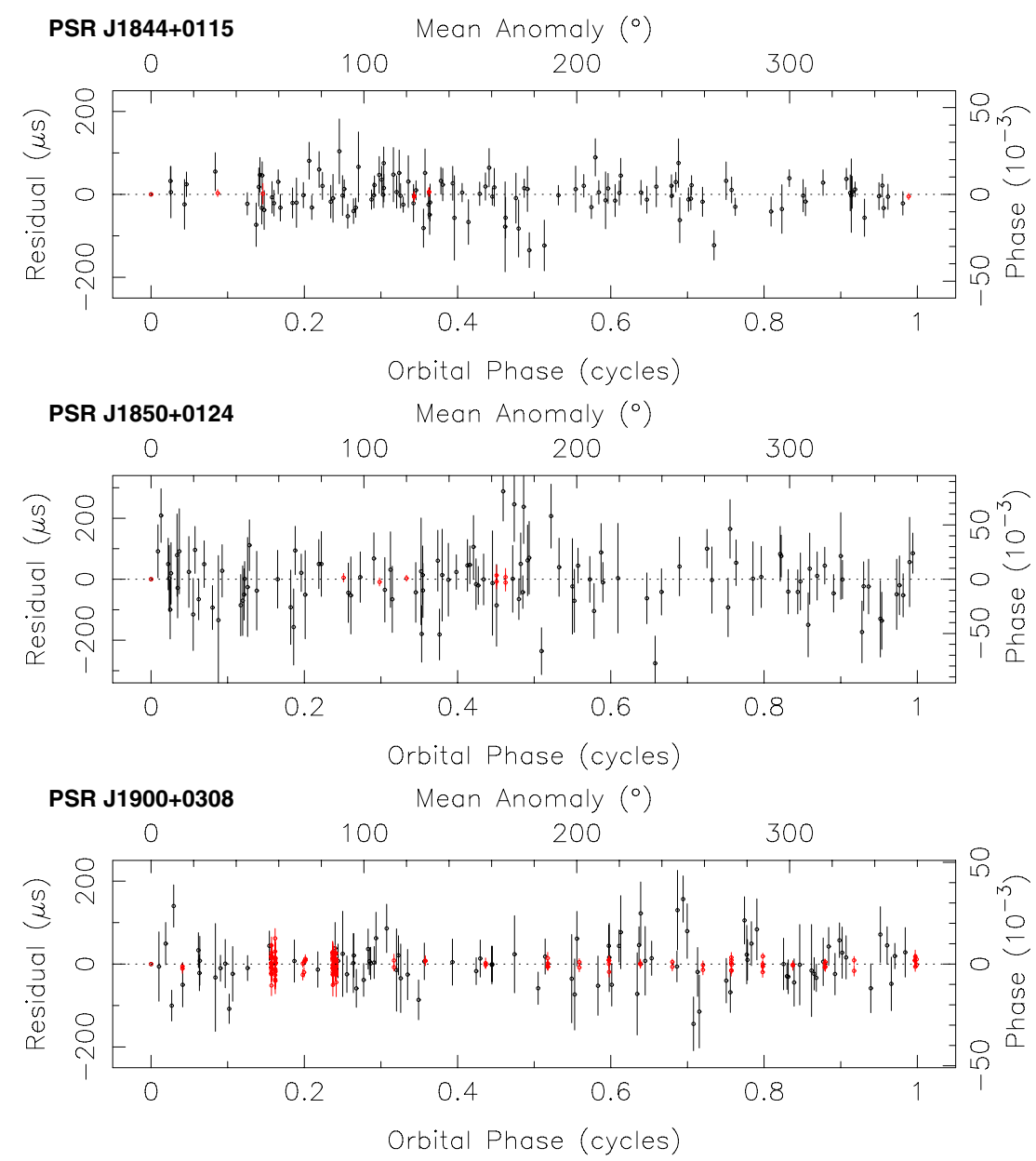

Figure 3. Timing residuals for three binary MSPs as a function of orbital phase. PSR J1944+2236 is isolated and is not shown. There are no systematic trends in the residuals. None of the three binaries show evidence of eclipsing effects which would be expected if the companions were extended, non-degenerate stars with large orbital inclination angles. This suggests that the companions are probably low-mass white dwarfs, though more massive companions with very small orbital inclinations cannot be ruled out.

in these estimates. For PSR J1944+2236, only an upper limit of $0.1 \mathrm{mJy}$ is quoted for the flux density since a value was not measured. This was chosen as a reasonable upper limit since this is the value measured for the next weakest pulsar, and if PSR J1944+2236 had this flux density, then it would have likely been measurable. Longer observations in the future using the ASP or different observations using a system with a wider bandwidth may be useful in obtaining RM measurements and a clearer indication of the polarization characteristics of these MSPs.

\section{DISCUSSION}

The inferred spin-down luminosities, surface magnetic field strengths, and characteristic ages of these four pulsars (Table 1) are typical of the values of most fully recycled pulsars. Likewise, the orbital properties of the three binaries presented here are typical, and they have low-mass companions $\left(M_{c} \sim 0.2 M_{\odot}\right)$ and low eccentricities ( $e \lesssim 3 \times 10^{-4}$ in all cases), which suggests that the systems are fully recycled with white dwarf companions.

The measured eccentricities of the three binaries can be compared to the relationship between the binary orbital period, $P_{b}$, and eccentricity, $e$, that was outlined by Phinney (1992) and Phinney \& Kulkarni (1994) for stable mass transfer from a Roche lobe filling red giant (see also Camilo et al. 2001 and Lorimer 2008). PSRs J1844+0115 and J1850+0124 have eccentricities that easily fall within the predicted range of the model for their orbital periods. PSR J1900+0308 has an eccentricity that is small compared to the predicted range but is still consistent with the model.

PSRs J1850+0124 and J1900+0308 may be useful for tests of the strong equivalence principle (SEP) and similar deviations from general relativity (Damour \& Schaefer 1991; Bell \& Damour 1996; Stairs et al. 2005; Gonzalez et al. 2011). The figure of merit for SEP tests is $P_{b}^{2} / e$, which for PSR J1850+0124 is large, $\gtrsim 10^{8}$ day $^{2}$. This places it in the same range as PSRs J1711-4322, J1933-6211, and J1853+1303 (see Table 1 of Gonzalez et al. 2011), which were among the best pulsars used to constrain violation of the SEP and the strong-field version of the parameterized post-Newtonian (PPN) parameter $\alpha_{3}$ in that paper (Will \& Nordtvedt 1972; Damour \& Esposito-Farèse 1992a).

PSR J1900+0308 has a very large lower limit for $P_{b}^{1 / 3} / e$, making it potentially a key pulsar for constraining the (strongfield) PPN parameter $\hat{\alpha_{1}}$ (Damour \& Esposito-Farèse 1992b; Bell et al. 1996). Historically, the pulsars used for this test have had orbital periods of just a few days, since these tend also to have the lowest orbital eccentricities. Unfortunately, the short orbital periods of the relevant pulsars make it hard to define a population of systems thought to have followed the same general evolutionary path; in particular, pulsars with white-dwarf companions and orbital periods under about four days cannot safely be assumed to follow the $P_{b}-m_{2}$ relation (Rappaport et al. 

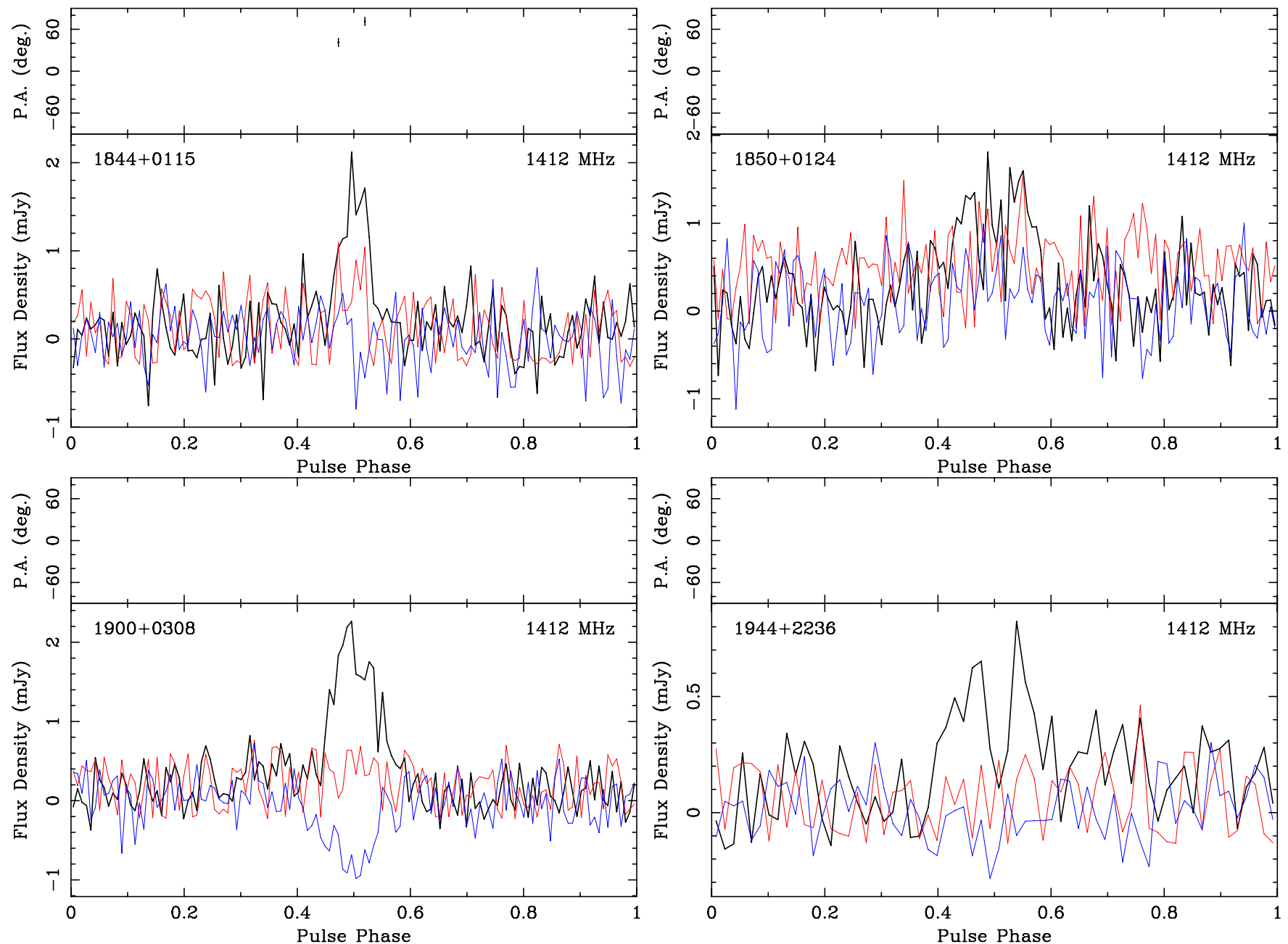

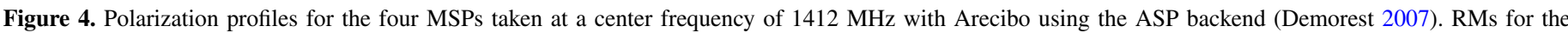

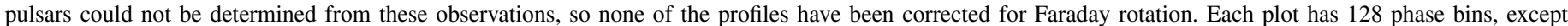

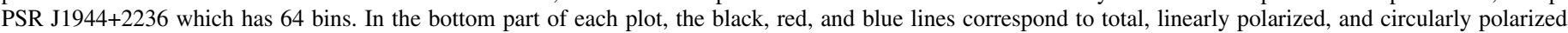

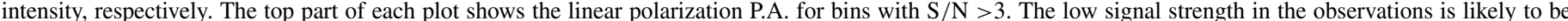
responsible for the lack of any significant linear polarization observed and any measurable P.A.s across the profiles.

1995; Tauris \& Savonije 1999; Thorsett \& Chakrabarty 1999). Since a well-defined population for which one can make reasonable evolution-based guesses at the pulsar and companion masses is desirable to mitigate against selection effects (Wex 2000), it has been difficult to define a suitable set of pulsars for use in the $\hat{\alpha_{1}}$ test. The existence of PSR J1900+0308, assuming it does follow the $P_{b}-m_{2}$ relation, opens the possibility of using this class of pulsar for the $\hat{\alpha_{1}}$ test. This will best be done once the proper motion and eccentricity are well measured for this system.

We are not currently able to measure proper motions for these pulsars. Making the assumption of a typical transverse speed of $\sim 100 \mathrm{~km} \mathrm{~s}^{-1}$ and a representative distance of $5 \mathrm{kpc}$, the

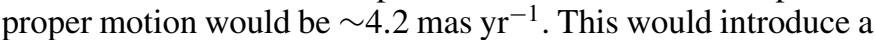
timing residual of $\sim 5 \mu$ s after one year (Shklovskii 1970), and the timing spans for these pulsars are relatively short $(\sim 2 \mathrm{yr}$ for three of the four pulsars; see Table 1). The relatively large rms values of the residuals (of order tens of $\mu \mathrm{s}$ ) preclude these objects from being useful in pulsar timing arrays in the search for gravitational waves. Given their large distances, their proper motions are likely to be small and difficult to measure given their timing precision, making it hard to accurately determine the kinematic contributions to $\dot{P}$ and $\dot{P}_{b}$.
We performed a test with the timing residuals in order to assess the role of red noise (if any) in the timing behavior of the four pulsars. The test we employed was the zero-crossing test, which measured the number of times the residuals change sign (cross zero) as a function of time (e.g., Deneva et al. 2012). The validity of this test is independent of the spacing of the individual timing points and corresponding residuals. The expected number of zero crossings of $N$ residuals if only white noise were present is $\left\langle Z_{W}\right\rangle=(N-1) / 2$, with an uncertainty in the mean of $\sigma_{Z_{W}}=\sqrt{(N-1)} / 2$. The presence of timing noise (red noise) will produce fewer crossings than expected. In all four cases, the observed number of residual crossings fell within the expected range $\left\langle Z_{W}\right\rangle \pm \sigma_{Z_{W}}$, indicating that red noise is not significant in the timing behavior of these pulsars at the current (rather poor) level of precision. We have also characterized the timing noise in these four MSPs using a relative timing noise parameter, $\zeta$, outlined by Shannon $\&$ Cordes (2010). $\zeta$ is the ratio of the observed timing residual value to a value expected from only timing noise. This latter parameter is determined using a scaling law (see their Equation (7)). The parameters used in the scaling were derived from measurements of canonical and MSPs (see Table 1 of Shannon \& Cordes 2010). For all four MSPs, we found that this ratio was $\zeta \gg 1$. This indicates that 


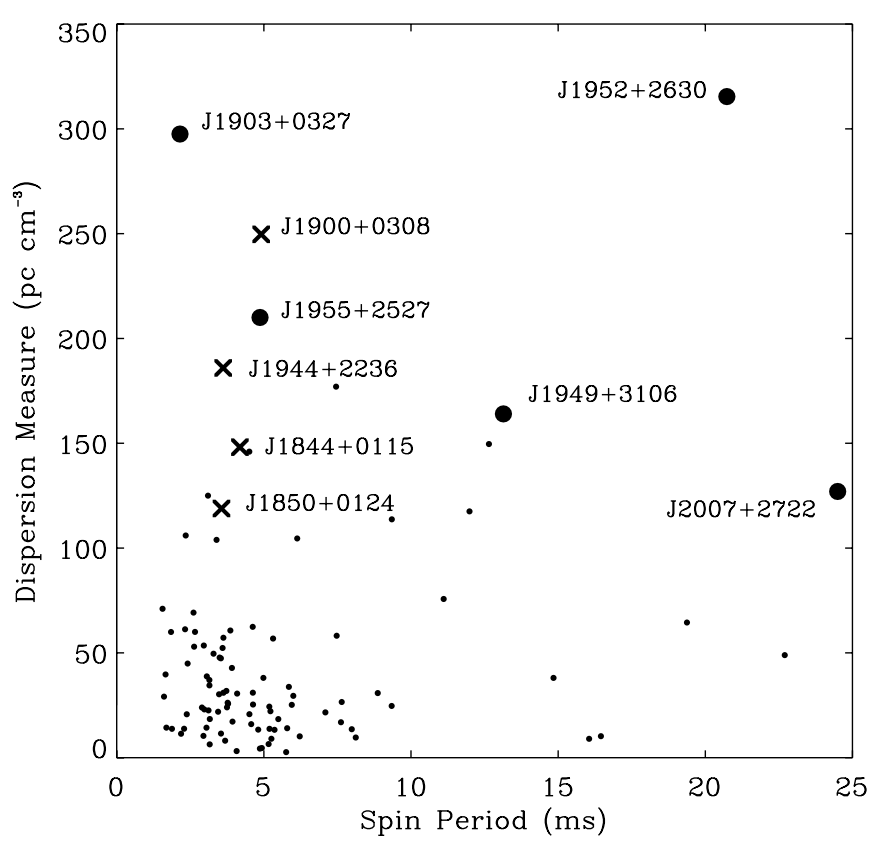

Figure 5. Dispersion measure vs. spin period for 90 Galactic field radio MSPs from the ATNF pulsar catalog (only pulsars with periods less than $25 \mathrm{~ms}$ and $\dot{P}<10^{-17}$ are plotted, and GC and radio-quiet pulsars are not included). These pulsars are plotted as dots. Also plotted are the four PALFA MSPs reported in this paper (crosses) and five other MSPs discovered by PALFA (filled circles) (Champion et al. 2008; Knispel et al. 2010, 2011; Deneva et al. 2012). All nine PALFA pulsars are labeled. It is clear that the PALFA survey is exploring new parameter space with the discovery of these pulsars. The DMs of these pulsars are among the highest that have been previously discovered for MSPs in the Galaxy.

white noise is dominating the residuals in all cases, and it is consistent with the results of the zero-crossing test above.

The relatively large distances to these pulsars are restrictive in terms of their potential for multi-wavelength follow-up, and, unsurprisingly, the SIMBAD database ${ }^{27}$ indicates that there are no optical or IR counterparts listed at the positions of two of the three binary pulsars. There is an unidentified IR source, IRAS 18421+0112, located 22" from PSR J1844+0115 which has a position uncertainty of $17^{\prime \prime}$ along the major axis of its position ellipse, but there is nothing listed in the 2MASS catalog at this location. It seems unlikely that this IRAS source is the binary counterpart of PSR J1844+0115, since the pulsar shows no evidence of eclipses or other variability/intermittency in the timing residuals that we might expect from a nondegenerate, extended companion (see Figure 3), and we see none of the timing jitter that is clearly present in the two known cases of these kinds of Galactic MSP systems that have been published to date (PSR J1023+0038, Archibald et al. 2009; and PSR J1723-2837, Crawford et al. 2010).

There are also no known X-ray or $\gamma$-ray counterparts in the HEASARC catalog at the four pulsar positions. This is not surprising since $E \lesssim 10^{34} \mathrm{erg} \mathrm{s}^{-1}$, and for this spindown luminosity range, the X-ray emission would be quite faint. To search for $\gamma$-ray pulsations from the four MSPs, we retrieved Fermi LAT data from the start of the mission up to 2012 February 6 using $1^{\circ}$ extraction radii centered on the pulsar positions. The event lists were filtered using the recommended cuts in maximum zenith angle $\left(100^{\circ}\right)$, event class (2), and photon energy (>100 MeV). The $\gamma$-ray events were folded using the

\footnotetext{
27 http://simbad.u-strasbg.fr/simbad/
}

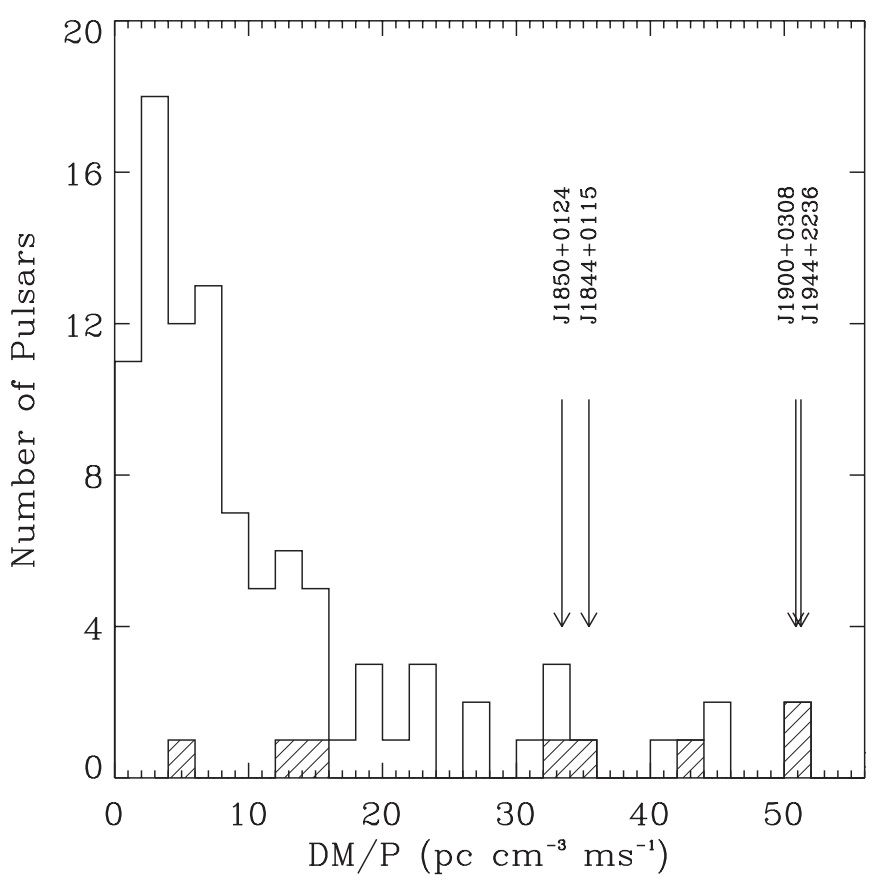

Figure 6. Histogram of $D M / P$ for 90 known field radio pulsars from the ATNF catalog and 8 PALFA MSPs (not including PSR J1903+0327) having $P<25 \mathrm{~ms}, \dot{P}<10^{-17}$. GC and radio-quiet pulsars are not included here. PSR J1903+0327 is not shown owing to its very large $\mathrm{DM} / P$ value of $138.4 \mathrm{pc} \mathrm{cm}^{-3} \mathrm{~ms}^{-1}$, which is far off the scale. The unshaded histogram shows all 98 pulsars, including the 8 PALFA pulsars. The shaded histogram shows the subset of eight PALFA MSPs. The four MSPs presented in this paper are indicated by arrows and are labeled. It is clear that the PALFA survey is finding fast distant pulsars that were undetectable in previous large-scale surveys.

Fermi plug-in for TEMPO $2^{28}$ and the radio ephemerides from Table 1 . In all instances, no statistically significant pulsations were detected. Repeating the analysis with a $>300 \mathrm{MeV}$ energy cut and extraction radii in the range $0.5-1^{\circ}$ also yielded no detections.

All four of these MSPs share the quality that they have very large DMs and DM-inferred distances. This is also a feature of the other MSPs that have been discovered by the PALFA survey. Previous large-scale surveys had poorer sensitivity to these kinds of MSPs owing to their lower observing frequencies and inadequate observing instrumentation (wider frequency channels and insufficient sampling rates). Figure 5 illustrates this with a plot of DM versus spin period for all 90 non-PALFA Galactic field radio pulsars in the ATNF pulsar catalog having $P<25 \mathrm{~ms}$ and $\dot{P}<10^{-17}$ (Manchester et al. 2005). ${ }^{29}$ This group of recycled pulsars does not include globular cluster (GC), radio quiet, or young pulsars. Also shown are the four MSPs described here and the five other PALFA MSPs that have been reported to date (Champion et al. 2008; Knispel et al. 2010, 2011; Deneva et al. 2012). It is clear that the PALFA MSPs occupy an area of the DM-period phase space where very few MSPs are currently known.

Figures 6 and 7 also illustrate the power of the PALFA survey for finding fast, distant MSPs. Both figures include the same set of data as shown in Figure 5. Figure 6 shows a histogram of the ratio DM $/ P$ (excluding PSR J1903+0327, since it falls well beyond the plot limits). The subset of PALFA MSPs is indicated with shaded parts of the histogram. The positions of

\footnotetext{
28 See http://fermi.gsfc.nasa.gov/ssc/data/analysis/user/Fermi_plug_doc.pdf.

29 http://www.atnf.csiro.au/research/pulsar/psrcat/
} 


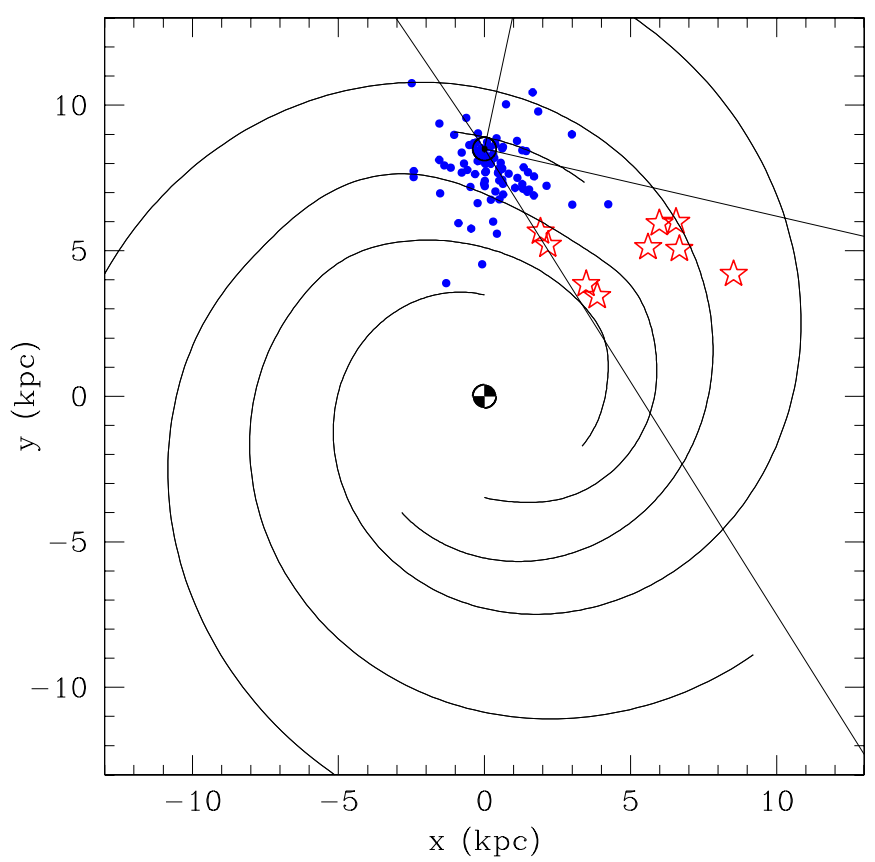

Figure 7. Overhead Galactic projection plot showing the nominal locations of 90 known field radio MSPs in the ATNF catalog with $P<25 \mathrm{~ms}$ and $\dot{P}<10^{-17}$ (blue dots) and 9 known PALFA MSPs (red stars) (Champion et al. 2008; Knispel et al. 2010, 2011; Deneva et al. 2012), including the 4 described in this paper. The nominal distances are inferred from the DMs and are uncertain by $\sim 30 \%$. The Sun is located at the center of the cluster of dots, and the Galactic center is at the origin. The black curves indicate locations of the Galactic spiral arms according to the NE2001 model of Cordes \& Lazio (2002). The cones indicated by the two pairs of straight lines indicate the regions of the Galactic plane that are visible from Arecibo. The PALFA MSPs are among the most distant non-cluster MSPs known.

the four MSPs presented here are indicated with arrows, all of which fall near the edge of the histogram. This plot demonstrates how we are exploring a larger DM $/ P$ parameter space with this survey. Figure 7 shows a Galactic projection plot of the same set of pulsars. The nominal locations of the PALFA MSPs in the Galactic plane are indicated by stars. Exploring the high DM $/ P$ parameter space is necessary for a full Galactic census of MSPs.

To demonstrate the potential of the PALFA survey in such a population analysis, we have carried out a preliminary investigation to model $\mathrm{DM} / P$ for Galactic MSPs. We used the freely available PSRPOP software package ${ }^{30}$ to carry out this work, and we generated simple "snapshot" models of the population which are normalized to reproduce the yield of 20 MSPs detected in the PMPS (e.g., Lorimer et al. 2006). The simulation procedure is described in that paper and also in Smits et al. (2009). In brief, we generated four different models (labeled A through D) of the present day MSP population which matched the number of MSPs detected by the PMPS, but produced a variety of different $\mathrm{DM} / P$ values and predicted yields for the PALFA survey. For the purposes of this work, we focused on changing assumptions about the spatial distribution of the underlying MSP population.

Model A assumes a Gaussian radial density profile with a standard deviation $\sigma_{R}=6.5 \mathrm{kpc}$, an exponential scale height with a mean of $h_{z}=500 \mathrm{pc}$, the log-normal $1400 \mathrm{MHz}$ pulsar luminosity function found by Faucher-Giguère \& Kaspi (2006), and the MSP period distribution and beaming model used in Smits et al. (2009). Model B is similar to Model A, with the

\footnotetext{
30 http://psrpop.sourceforge.net
}

Table 2

MSP Population Simulation Results for Four Models

\begin{tabular}{lcccc}
\hline \hline Sample & $N_{\text {PMPS }}{ }^{\mathrm{a}}$ & $N_{\text {PALFA }}{ }^{\mathrm{a}}$ & $\mathrm{DM} / P_{\text {PMPS }^{\mathrm{b}}}$ & $\mathrm{DM} / P_{\text {PALFA }}{ }^{\mathrm{b}}$ \\
\hline Observed & 20 & $>15$ & 8 & 33 \\
Model A & 20 & 40 & 14 & 56 \\
Model B & 20 & 15 & 9 & 7 \\
Model C & 20 & 37 & 11 & 113 \\
Model D & 20 & 42 & 16 & 27 \\
\hline
\end{tabular}

Notes. The four models and their parameters are described in the text in Section 3.

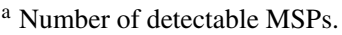

b Median DM $/ P$ value of detectable MSPs.

exception that the radial density is uniform (i.e., a constant surface density) throughout a disk of radius $25 \mathrm{kpc}$. Models $\mathrm{C}$ and D both follow Model A, but with $h_{z}$ set to 250 and $750 \mathrm{pc}$, respectively. Further models should be explored in a subsequent analysis, but for the purposes of the present work, the above choices demonstrate the importance of $\mathrm{DM} / P$ as a diagnostic for population studies.

The results of our simulations are shown in Table 2, where we tabulate, along with the observed sample, the numbers of detectable MSPs along with the median DM $/ P$ found in each simulation. By definition, due to the way we normalize the simulations, the number of detected MSPs in the PMPS, $N_{\text {PMPS, }}$ is 20. For models A through D, we tabulated the predicted number of MSPs detectable in the PALFA survey, $N_{\text {PALFA }}$. The median $\mathrm{DM} / P$ values for each simulation can be compared with those currently observed in the survey samples. As can be seen, there is a tremendous variation in the predicted values, and the median $\mathrm{DM} / P$ depends strongly on the choice of scale height and radial distribution. Model B appears to underestimate both the observed number of PALFA MSPs (assuming we find more as the survey proceeds) as well as the median $\mathrm{DM} / P$ value. Model $\mathrm{C}$ seems to overestimate the median $\mathrm{DM} / P$ value. Models $\mathrm{A}$ and $\mathrm{D}$ are most consistent with our observations to date, suggesting that a large-scale height $h_{z}$ and a Gaussian radial profile density are favored.

\section{CONCLUSIONS}

With its high time and frequency resolution and relatively high observing frequency, the PALFA survey is sensitive to Galactic field MSPs at large distances and DMs. The survey has so far discovered a total of 15 MSPs with $P<25$ ms, 14 of which have DM $>100 \mathrm{pc} \mathrm{cm}^{-3}$. Four of these PALFA MSPs (PSRs J1844+0115, J1850+0124, J1900+0308, and J1944+2236) are presented here with phase-coherent timing solutions. As an ensemble, the PALFA MSP discoveries show the ability of PALFA to extend the volume of MSP discovery space to relatively high DMs. This is the first step toward a nearly complete census of the Galactic MSP population, which will be possible with the SKA.

The Arecibo observatory is operated by SRI International under cooperative agreement with the National Science Foundation (AST-1100968) and in alliance with Ana G. MendezUniversidad Metropolitana, and the Universities Space Research Association. This work was also supported by NSF grant AST0807151, by NSERC Discovery Grants, by FQRNT via the Centre de Recherche Astrophysique du Quebec, by CIFAR, by CANARIE, by Compute Canada, by the Canada Foundation for Innovation, and a Killam Research Fellowship. V.M.K. 
holds the Lorne Trottier Chair in Astrophysics and Cosmology and a Canadian Research Chair in Observational Astrophysics. J.W.T.H. acknowledges funding from an NWO Veni Fellowship. B.K. gratefully acknowledges the support of the Max Planck Society. P.L. was partly funded by an NSERC PGS scholarship and an IMPRS fellowship. P.F. gratefully acknowledges the financial support by the European Research Council for the ERC Starting Grant BEACON under contract No. 279702.

\section{REFERENCES}

Archibald, A. M., Stairs, I. H., Ransom, S. M., et al. 2009, Science, 324, 1411 Bell, J. F., Camilo, F., \& Damour, T. 1996, ApJ, 464, 857

Bell, J. F., \& Damour, T. 1996, Class. Quantum Grav., 13, 3121

Bhat, N. D. R., Cordes, J. M., Camilo, F., Nice, D. J., \& Lorimer, D. R. 2004, ApJ, 605,759

Camilo, F., Lorimer, D. R., Freire, P., Lyne, A. G., \& Manchester, R. N. 2000, ApJ, 535, 975

Camilo, F., Lyne, A. G., Manchester, R. N., et al. 2001, ApJ, 548, L187

Carilli, C. L., \& Rawlings, S. 2004, New Astron., 48, 979

Champion, D. J., Ransom, S. M., Lazarus, P., et al. 2008, Science, 320, 1309

Cordes, J. M., Freire, P. C. C., Lorimer, D. R., et al. 2006, ApJ, 637, 446

Cordes, J. M., \& Lazio, T. J. W. 2002, arXiv:astro-ph/0207156

Crawford, F., et al. 2010, BAAS, 42, 604

Damour, T., \& Esposito-Farèse, G. 1992a, Class. Quantum Grav., 9, 2093

Damour, T., \& Esposito-Farèse, G. 1992b, Phys. Rev. D, 46, 4128

Damour, T., \& Schaefer, G. 1991, Phys. Rev. Lett., 66, 2549

Demorest, P. B. 2007, PhD thesis, Univ. California, Berkeley

Demorest, P. B., Pennucci, T., Ransom, S. M., Roberts, M. S. E., \& Hessels, J. W. T. 2010, Nature, 467, 1081

Deneva, J., Freire, P. C. C., Cordes, J. M., et al. 2012, ApJ, submitted

Dowd, A., Sisk, W., \& Hagen, J. 2000, in ASP Conf. Ser. 202, IAU Colloq. 177: Pulsar Astronomy-2000 and Beyond, ed. M. Kramer, N. Wex, \& R. Wielebinski (San Francisco, CA: ASP), 275

Faucher-Giguère, C.-A., \& Kaspi, V. M. 2006, ApJ, 643, 332
Freire, P. C. C., Bassa, C. G., Wex, N., et al. 2011, MNRAS, 412, 2763

Gonzalez, M. E., Stairs, I. H., Ferdman, R. D., et al. 2011, ApJ, 743, 102

Hessels, J. W. T., Ransom, S. M., Stairs, I. H., et al. 2006, Science, 311, 1901

Hobbs, G., Archibald, A., Arzoumanian, Z., et al. 2010, Class. Quantum Grav., 27,084013

Hobbs, G. B., Edwards, R. T., \& Manchester, R. N. 2006, MNRAS, 369, 655

Hotan, A. W., van Straten, W., \& Manchester, R. N. 2004, PASA, 21, 302

Jenet, F. A., Hobbs, G. B., Lee, K. J., \& Manchester, R. N. 2005, ApJ, 625, L123

Johnston, H. M., \& Kulkarni, S. R. 1991, ApJ, 368, 504

Keith, M. J., Jameson, A., van Straten, W., et al. 2010, MNRAS, 409, 619

Knispel, B., Allen, B., Cordes, J. M., et al. 2010, Science, 329, 1305

Knispel, B., Lazarus, P., Allen, B., et al. 2011, ApJ, 732, L1

Lange, C., Camilo, F., Wex, N., et al. 2001, MNRAS, 326, 274

Lattimer, J. M., \& Prakash, M. 2007, Phys. Rep., 442, 109

Lorimer, D. R. 2008, Living Rev. Rel., 11, 8

Lorimer, D. R., Faulkner, A. J., Lyne, A. G., et al. 2006, MNRAS, 372, 777

Manchester, R. N., Hobbs, G. B., Teoh, A., \& Hobbs, M. 2005, AJ, 129, 1993

Manchester, R. N., Lyne, A. G., Camilo, F., et al. 2001, MNRAS, 328, 17

Phinney, E. S. 1992, Phil. Trans. R. Soc. A, 341, 39

Phinney, E. S., \& Kulkarni, S. R. 1994, ARA\&A, 32, 591

Ransom, S. M. 2001, PhD thesis, Harvard Univ.

Ransom, S. M., Eikenberry, S. S., \& Middleditch, J. 2002, AJ, 124, 1788

Ransom, S. M., Ray, P. S., Camilo, F., et al. 2011, ApJ, 727, L16

Rappaport, S., Podsiadlowski, P., Joss, P. C., Di Stefano, R., \& Han, Z. 1995, MNRAS, 273, 731

Shannon, R. M., \& Cordes, J. M. 2010, ApJ, 725, 1607

Shklovskii, I. S. 1970, SvA, 13, 562

Smits, R., Kramer, M., Stappers, B., et al. 2009, A\&A, 493, 1161

Stairs, I. H., Faulkner, A. J., Lyne, A. G., et al. 2005, ApJ, 632, 1060

Tauris, T. M., \& Savonije, G. J. 1999, A\&A, 350, 928

Thorsett, S. E., \& Chakrabarty, D. 1999, ApJ, 512, 288

Wex, N. 2000, in ASP Conf. Ser. 202, IAU Colloq. 177: Pulsar Astronomy-2000 and Beyond, ed. M. Kramer, N. Wex, \& N. Wielebinski (San Francisco: ASP), 113

Will, C. M., \& Nordtvedt, K., Jr. 1972, ApJ, 177, 757 